\title{
Stress hilft nur den Viren
}

Wer gestresst ist, wird häufiger krank. Was viele Ärzte aus der Praxis wissen, beschäftigt das relativ neue Forschungsgebiet der Psychoneuroimmunologie. Es klärt den Einfluss der Psyche auf das Immunsystem auf. Forschungen, die an der ETH Zürich initiiert wurden, zeigen, dass sozialer Stress akut und die posttraumatische Belastungsstörung langfristig die Abwehrkräfte des Körpers beeinflussen.

Es war erstaunlich, was Anatomen über den Thymus schon zu Beginn des letzten Jahrhunderts bemerkten. Obduzierten sie Unfalltote, die plötzlich aus dem gesunden Leben gerissen wurden, fanden sie in der Regel den Thymus ordentlich gross entwickelt vor. Bei der Obduktion von an Krankheiten Dahingerafften war er in der Regel viel kleiner. «Damals erschien es erstmals als Möglichkeit, dass Stress, wie er durch Krankheiten auf den Körper ausgeübt wird, direkt auf das Immunsystem wirkt», sagt Harald Engler, der sich an der ETH Zürich in der Arbeitsgruppe von Professor Manfred Schedlowski mit der Auswirkung von psychischen Belastungen auf das Abwehrsystem beschäftigt hat. Jetzt ist die Gruppe an der Universität Essen tätig, wobei noch einige Arbeiten in Zürich durchgeführt werden.

«Die höhere Anfälligkeit von Depressiven für Infektionen lässt sich auf eine biochemische Wirkungskette zurückführen, die über Hypothalamus-Hypophysen und Nebennierenachse läuft.»

Allerdings waren die Kenntnisse über das Immunsystem rudimentär; man glaubte noch, dass die Lymphozyten zur Ernährung anderer Zellen dienten, über die Funktion der Milz herrschte Unklarheit und die Biochemie von Stress war erst in den Anfängen untersucht. Kein Wunder, dass die Teildisziplinen Psychologie, Endokri- nologie und Immunologie getrennt versuchten, Fortschritte auf ihren Feldern zu erzielen. In den letzten 15-20 Jahren hat sich aber die fächerübergreifende Forschung über den Zusammenhang von Psyche, Immunsystem und Krankheiten verstärkt. Mittlerweile sind die Schlussfolgerungen aus den Ergebnissen der Psychoneuroimmunologie unbestritten, dass sich psychische Belastungen tatsächlich direkt auf das Immunsystem auswirken und damit wahrscheinlich auch einen entscheidenden Einfluss auf die Entstehung von Krankheiten haben. Wissenschafter konnten eine Reihe von kausalen Zusammenhängen aufklären. Aber nicht nur der afferente Weg, die Wirkung von der Psyche auf das Abwehrsystem, konnte in einer grossen Zahl von Untersuchungen gezeigt werden. Wahrscheinlich ist auch der efferente Weg: Das Immunsystem beeinflusst die Psyche. Wissenschafter glauben so zum Beispiel den Placeboeffekt erklären zu können. «Man hat diese Zusammenhänge lange nicht gesehen, weil man sich zu sehr auf das eigene Fachgebiet konzentriert und das Gesamtsystem aus den Augen verloren hat», sagt Engler.

Die höhere Anfälligkeit von Depressiven für Infektionen lässt sich auf eine biochemische Wirkungskette zurückführen, die über HypothalamusHypophysen und Nebennierenachse läuft. Die Unterdrückung des Immunsystems wird auch durch die er- höhte Sekretion von Glucocorticoiden erklärt, die über verschiedene Mechanismen und Zwischenschritte die Produktion von Immunglobulinen hemmen.

\section{Angst vor dem Chef macht krank}

Sozialer Stress gehört in der modernen Arbeitswelt wahrscheinlich zu den Faktoren, die einem Menschen am meisten zusetzen. Leistungsd ruck, das Einordnen in Hierarchien oder etwa Unsicherheit sind Belastungen, die zum Beispiel zu einem Burn-out führen können, wenn sie über eine längere Zeit andauern. Wissenschafter haben versucht, die Auswirkung von sozialem Stress auf das Immunsystem zu verstehen. Dabei benutzen sie Mäuse; ihre Versuchsanordnung ist die der «sozialen Disruption». Jeden Tag wird in die Mäusegruppe ein jeweils neues, also unbekanntes dominantes Tier gesetzt. Das stellt die Rangordnung auf den Kopf, Kämpfe werden ausgetragen, sozial niedrige Mäuse kommen unter Druck und die oberen müssen ihren Staus verteidigen. «Das ist eine Situatiaton, in der die gesamten Sozialbeziehungen sich auflösen und neu geformt werden müssen», sagt Engler. Und dies jeden Tag.

In einer Reihe von Versuchen haben er und seine Mitarbeiter sich mit der Reaktion des Immunsystems beschäftigt, das durch den täglichen

\section{KARGER}

Fax +49 7614520714 
Chefwechsel innerhalb der Mäusegruppe sehr in Mitleidenschaft gezogen war. Die Veränderungen zeigten sich darin, dass verschiedene Immunzellen unempfindlicher gegenüber der Steuerung durch den Körper wurden. Auswirkungen von sozialen Herausforderungen beeinflussen die Glucocorticoid-Antwort von Immunzellen bei Mäusen. Bei den wiederholt gestressten Mäusen reagierten bestimmte Immunzellen weniger stark auf die antiinflammatorische Wirkung der Glucocorticoide. Sie werden unempfindlicher für die Signale, die von ihnen ausgehen. Bei Makrophagen sprechen die Forscher sogar von einer Resistenz gegen die Anweisungen, mit denen der Körper sie durch die Glucocorticoide steuert. Im Körper der gestressten Tiere stieg der Titer von proinflammatorischen Zytokinen an; ein deutliches Zeichen, dass das Immunsystem mit einer Infektion nicht zurechtkommt. Andere Zellen des Immunsystems, die CD11b+ Zellen, die nicht auf die Glucocorticoide ansprechen, nahmen in ihrer Anzahl in der Milz stark zu. Der Stress durch die sozialen Auseinandersetzungen führte

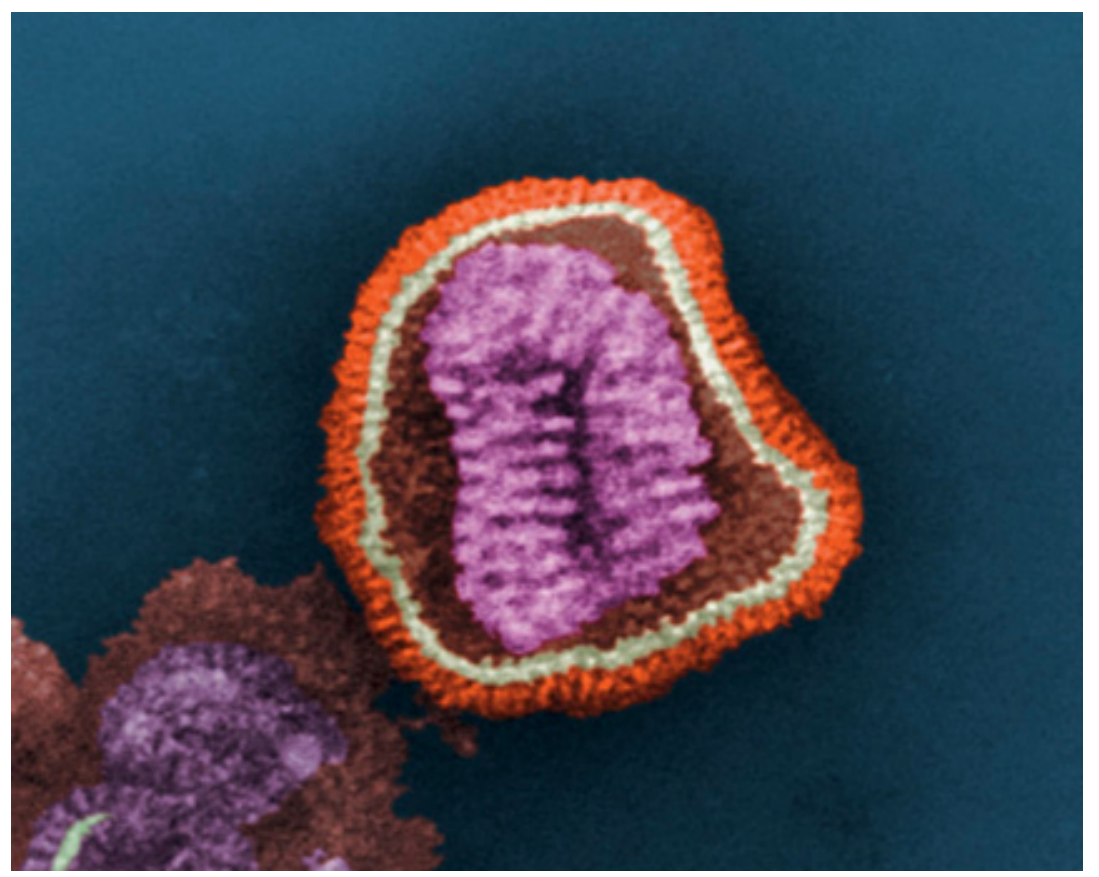

Abb. 1. Virus aus der Gattung Influenzavirus in einer Transmissionselektronenmikroskopie-Aufnahme. zu Veränderungen der Verteilung von Immunzellen im Knochenmark, im peripheren Blut und in der Milz. «Mäuse, die häufiger sozialer Disruption ausgesetzt waren, waren insgesamt weniger sensitiv gegen eine Immunantwort», sagt Engler. Mit diesen Ergebnissen haben die Forscher einen Teil der Wirkungskette aufklären können, welchen Einfluss Stress auf das Immunsystem hat. Bislang war lediglich bekannt, dass Stresshormone immunsuppressiv wirken.

Auch auf der Ebene der Infektionen selbst konnten die Forscher Unterschiede zwischen Mäusen finden, die sozial gestresst waren, und solchen, die in einem stabilen Umfeld lebten. Nach wiederholtem Durcheinanderwirbeln der Sozialstruktur wurden im Organismus der Mäuse deutlich mehr Bakterien gefunden, die wahrscheinlich über die Lymphknoten eingewandert waren. In einer nächsten Phase werden sich die Wissenschafter in der Arbeitsgruppe von Manfred Schedlowski auch mit der direkten Antwort des Körpers auf Infekte beschäftigen. Harald Engler plant, dabei eine Kontrollgruppe und sozial gestresste Tiere mit einem Virus $\mathrm{zu}$ infizieren. $\mathrm{Ob}$ sich das erwartete Ergebnis wirklich finden lässt, wird die Zukunft zeigen.

\section{Traumatische Erfahrungen wirken lange}

Bei Menschen, die schlimme Ereignisse zu verarbeiten haben und unter einem posttraumatischen Schock (PTSD) leiden, schlägt das Immunsystem über die Stränge. Zu den Betroffenen gehören Menschen, die aus Kriegsgebieten geflohen sind, Opfer von Gewalt geworden sind oder sie mit ansehen mussten. Oft leiden auch Soldaten oder Polizisten, Rettungssanitäter oder Ärzte, die durch schreckliche Bilder überfordert wurden, unter den Eindrücken ihrer Erlebnisse. Schon lange ist bekannt, dass posttraumatischer Stress mit verschiedenen somatischen Beschwerden und Krankheiten verbunden ist, wobei die genaue Verkettung zwischen dem Stress und diesen somatischen Erscheinungen aber unklar ist.

«Mittlerweile sind die Schlussfolgerungen aus den Ergebnissen der Psychoneuroimmunologie unbestritten, dass sich psychische Belastungen tatsächlich direkt auf das Immunsystem auswirken und damit wahrscheinlich auch einen entscheidenden Einfluss auf die Entstehung von Krankheiten haben.»

Eine ganze Reihe von Arbeitsgruppen beschäftigt sich mit der immunologischen Auswirkung von solchem Stress. Dabei hat die Arbeitsgruppe eine wichtige Rolle gespielt. Klar ist: Der posttraumatische Stress schwächt das Immunsystem, und zwar auf lange Sicht. Wie sich die Schreckensbilder im Gehirn eingebrannt haben, haben sie auch ihre tiefen Spuren im Immunsystem hinterlassen. Wissenschafter untersuchen dabei verschiedene Parameter des Immunsystems bei traumatisierten Menschen, wie etwa die Anzahl verschiedener Lymphozyten, 
aber auch die Konzentration von Corticosteroiden. In einer Untersuchung bei 19 Menschen mit einem PTSD fanden die Forscher um Harald Engler, dass eine Reihe von Lymphozytenwerten bei ihnen deutlich reduziert war. Die Anzahl der CD8-T-Lymphozyten war um ein Drittel reduziert, die An- zahl der CD3- und Memory-Lymphozyten um ein Viertel bzw. ein Drittel erhöht. Die traumatisierten Menschen hatten eine um die Hälfte verringerte Konzentration von regulatorischen T-Zellen - und dies selbst Monate nach den Ereignissen, denen sie ausgesetzt waren. Die Forscher sprechen von einer tiefgreifend veränderten Zusammensetzung der peripheren T-Zellen-Population. Diese könnte für eine reduzierte Immunantwort verantwortlich sein, die auch erklären könnte, weshalb PTSD-Patienten eine erhöhte Anfälligkeit für Infektionen und Entzündungen haben.

\section{Der Wirkmechanismus der Psyche auf das Immunsystem}

Hormone, Transmitter und Peptide wirken auf die Zellen des Immunsystems. Durch sie beeinflussen psychische Faktoren das Abwehrsystem des Menschen wahrscheinlich stark und machen ihn anfällig für Infektionen. Von einem umfassenden Modell der Wirkung ist die Forschung zwar noch weit entfernt, doch die kausalen Wirkungen sind plausibel.

Das Immunsystem wird von einer Reihe von Hormonen, Neurotransmittern und Neuropeptiden beeinflusst, deren Ausstoss in vielen Fällen von der psychischen Belastung des Organismus abhängt. Ein umfassendes Modell, wie die verschiedenen Faktoren zum Beispiel bei akutem oder chronischem Stress auf das Immunsystem wirken, gibt es allerdings noch nicht. Stattdessen liegen aus den letzten 15-20 Jahren eine ganze Reihe von Untersuchungen vor, deren Ergebnisse deutlich machen, wie sehr das Immunsystem unter endokrinem Einfluss steht. Diese Mosaiksteine lassen ahnen, wie stark der Einfluss der Psyche auf die körperliche Gesundheit ist.

Bei akutem psychischem und physischem Stress werden zum Beispiel die Katecholamine Adrenalin und Noradrenalin, deren Produktion durch das sympathische Nervensystem gesteuert wird, im Nebennierenmark freigesetzt. Sie sorgen dafür, dass der Körper auf den Stress mit einer «fight or flight»Reaktion angemessen reagieren kann. Unter Stress werden alle nicht lebenswichtigen Funktionen reduziert und diejenigen besonders stark aktiviert, die für Flucht oder Verteidigung nötig sind. Er sorgt dafür, dass die Verdauung gehemmt wird, die Gefässe erweitert und Sauerstoff und Glukose für eine mögliche körperliche Belastung bereitgestellt werden. Auf die Immun- zellen wirken die vom Stress freigesetzten Katecholamine über Rezeptoren auf der Zelloberfläche und eine danach über die Aktivierung von «second messengern» ausgelöste intrazelluläre Antwort. Adrenalin und Noradrenalin binden an zwei Adrenorezeptoren, die sich auf allen Lymphozytentypen befinden. Untersuchungen deuten darauf hin, dass die beiden Katecholamine dafür verantwortlich sind, dass die Aktivität der Killerzellen ansteigt und sie vermehrt in das periphere Gewebe transportiert werden. Die Produktion von T-Lymphozyten wird durch die beiden Katecholamine allerdings gehemmt.

Über die Hypothalamus-Hypophysen-Achse wird zum Beispiel die Produktion von Prolaktin, Thyreotropin, des Wachstumshormons, des Follikelstimulierenden und des luteinisierenden Hormons gesteuert, die ihrerseits Aktivität und Produktion der verschiedenen Lymphozyten beeinflussen. Glucocorticoide, die in der Nebennierenrinde produziert werden, wirken, wenn sie als Medikamente über einen längeren Zeitraum gegeben werden, immunsuppressiv. Sie unterdrücken die Produktion von Zytokinen, die als Botenstoffe eine wichtige Rolle in der Kaskade der Immunantwort spielen und für die Steuerung der Immunreaktion verantwortlich sind. Durch die Wirkung der Glucocorticoide be- dingte geringere Aktivität der Zytokine wird die Antwort des Abwehrsystems schwächer. Allerdings gibt es Befunde, die darauf hindeuten, dass Cortisol, das ebenfalls ein Glucocorticoid ist, regulierend in das Immunsystem eingreifen kann. Es scheint, dass es einen modulierenden Effekt auf das Immunsystem hat und Extremreaktionen verhindert.

Einen sehr direkten Effekt hat Endorphin, das auch als Glückshormon bezeichnet wird. Es wird bei grosser körperlicher Belastung ausgeschüttet und führt zu emphatischen Zuständen, wie etwa dem Hochgefühl nach einer längeren Joggingtour, das auch als «runner's high» bezeichnet wird. Es wird von der Hypophyse und dem Zentralen Nervensystem ausgeschüttet, bindet an Opioid-Rezeptoren und hat eine Wirkung, die dem von Morphin ähnlich ist. Auch Immunzellen tragen solche Rezeptoren an ihrer Oberfläche. Wenn Endorphine an sie ankoppeln, kann es eine Reihe von Immunfunktionen regulieren. Die Produktion von Lymphozyten wird in Abhängigkeit von der Endorphin-Konzentration gesteigert. Die genaue Wirkungskaskade ist allerdings noch nicht geklärt. Für eine ganze Reihe von anderen Hormonen sind ebenfalls Rezeptoren auf der Oberfläche von Immunzellen gefunden worden. (rfi) 